\title{
Improved K-means Clustering Color Segmentation for Road Perception
}

\author{
Lei ZHOU ${ }^{1, a}$, Yanjun ZHANG ${ }^{1, b}$, Danwen PENG ${ }^{2, ~ c}$, Dimin WU ${ }^{1, d}$ \\ ${ }^{1}$ Department of Ground artillery, Wuhan Ordnance Noncommissioned Officer Academy, Wuhan, \\ 430075, China
}

${ }^{2}$ PLA 95007 Unit, Guangzhou, 510095, China

aemail:kongjzhlei@163.com, bemail:digua5557@sina.com, 'email:jidan311@163.com , demail:semicopy@163.com

Keywords: Road Perception; Color Model; K-means Clustering Segmentation; B-splines Curve Model.

\begin{abstract}
A modified road perception algorithm is presented based on the color image clustering segmentation. According to the comparison of color spaces' uniformity and integrity, an improved K-means clustering algorithm is proposed to segment color images in the space LAB. Firstly, the target area contains road which is gained in images class using the connected domain labeling algorithm. Then, credible road edge points can be obtained in response to alternate-line sampling labeled region of images and assuming the constant of road width consequently. By establishing the B-splines curve model to fit road shape, the algorithm adopts the least square method used to search the optimal control points of splines curve to identify the road boundaries.
\end{abstract}

\section{Introduction}

Color images [1] [2] are rich in color and texture information, which segmentation is a very complex problem. There are few algorithms that approach or reach the artificial segmentation results. Different color models can be used to represent image color, meanwhile color segmentation algorithms are many different [3]. In order to ensure color images segmentation satisfactorily, not only we must choose an appropriate color space, but also adopt segmentation strategies and methods suitable for this space.

Unstructured road and especially field road are provided with complex structure and changing shape[4] [5]. Road models must match the road construction of the actual environment which directly can affect the accuracy of road detection. B-splines curve [6] [7] is flexible and localized Compared to other curves when fitting the actual models. B-splines curve can fit any shape if it is given sufficient control, meanwhile partial modification of the splines will not affect other parts of the curve.

\section{Color Models Selection}

The three components in RGB color space are closely related and hue is sensitivity to the change of component numerical so that RGB color space is not suita le for the $\mathrm{K}$ means clustering algorithm. Experiment shows the transformation from YIQ space, $\mathrm{YC}_{b} \mathrm{C}_{\mathrm{r}}$ space into the RGB space is linear, therefore, the correlation of each variable in the YIQ space, $\mathrm{YC}_{b} \mathrm{C}_{r}$ space is strong, not suitable for the clustering algorithm [8]. Known from other color models conversion relationship, three variables in the $\mathrm{LAB}$ color space are retained information of three variables in the RGB color space, but three variables in the HSV color space are retained only part of the variables' information in the RGB color space. Figure1 is displayed with one road image in the three color space Figure 1(a) is a road image in the RGB model Figure 1(b) is a road image in the HSV model Figure 1(c) is a road image in the $\mathrm{LAB}$ model. 


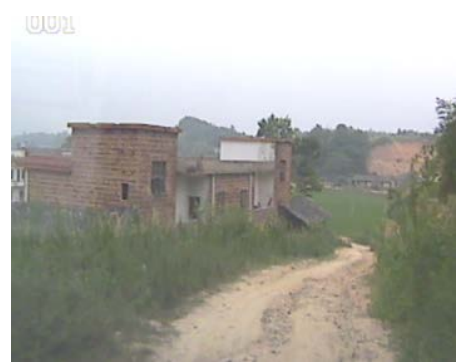

(a) RGB model

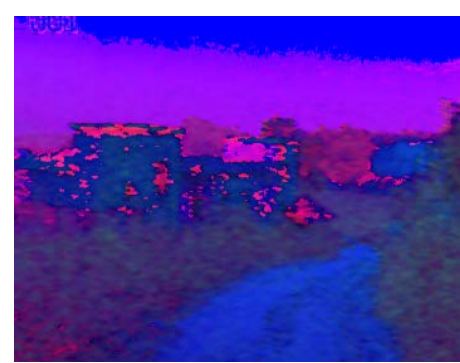

(b) HSV model

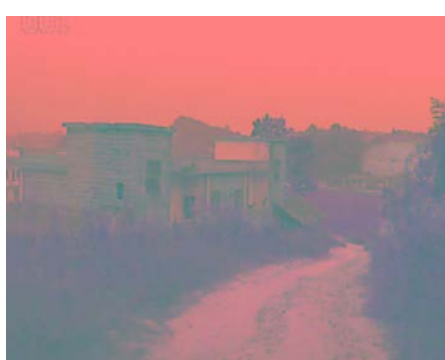

(c) LAB model

Fig.1. The same image is showed in three color space

HSV color space can reflect three kinds of features huev saturation and brightness of the color but it is not uniform color space by perception, namely the change of distance on the space is not entirely representative of the color vision. The LAB model has a wide color gamut, not only contains all the color gamut of RGB, but also solves the problem of unevenly distributed color in the RGB space. So this paper chooses color segmentation of road images in LAB space.

\section{Color Image Segmentation}

We can evaluate the number of categories according to color theory and threshold theory. Firstly we transform the collected road color image into HSV space, and then transform the hue component into a gray image in order to calculate the histogram [9], finally evaluate the number of clustering directly according to the number of the effective wave crest of histogram. The effective wave crest is defined as the number of the area that is more than $1 / 5$ numbers of entire pixels under the curve. If the number of effective wave crest is equal to 2 , the number of categories is equal to 3 . If the number of effective wave crest is larger than 2 , the number of categories is equal to 4 . If the number of effective wave crest is less than 2, the number of categories is equal to 2 . We take a color image Figure3 (a) as an example and Figure2 is the $\mathrm{H}$ component histogram. Because the effective wave number is 3 , the number of categories is set to 4 . Figure3 (c) (e) are categories images after K-means clustering segmentation.

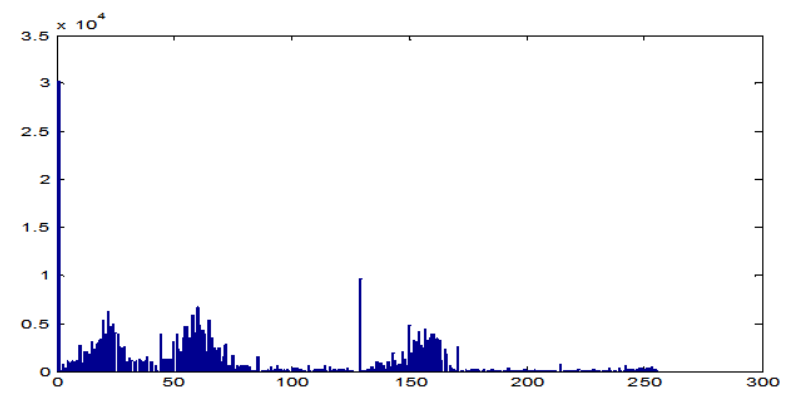

Fig.2. Histogram of hue component

The specific steps of improved K-means clustering algorithm are as follows:

Step1: Reading a 24 bit true color image (assuming the image size is $m \times n \times 3$ ), the color image is converted from RGB color space to LAB color space.

Step2: Rank pixels in AB color space into a matrix $M(m \times n \times 2)$ after extraction of AB spatial information in LAB color space.

Step3: The data in the matrix is divided into $k(k=2,3,4$, determined by the above method) using k-means clustering algorithm and obtain the cluster center and clustering results automatic assignment of pixels in each category is $1,2,3, \ldots, k$ ). The clustering center is matrix $k \times 2$.

Step4: Transform the clustering result into a matrix $m \times n$ and generate the image as shown in Figure 3(b).

Step5: On the basis of step4, the pixels do not belong to the class $k$ the assignment of which is 0 , the pixels belong to the class $k$ assignment of which is the original pixel value. Different categories generated from different $k$ images of image are as shown in Figure3 (c) (f).

Step6: In different $k$ image, we acquire the optimal solution to obtain the target area. 


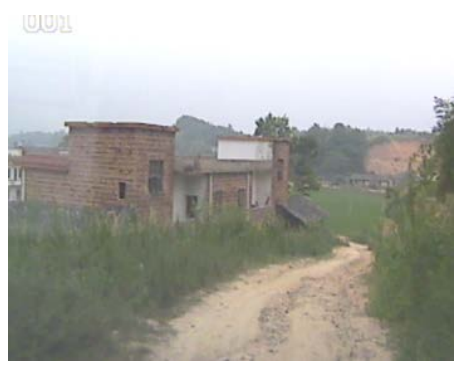

(a) The original image

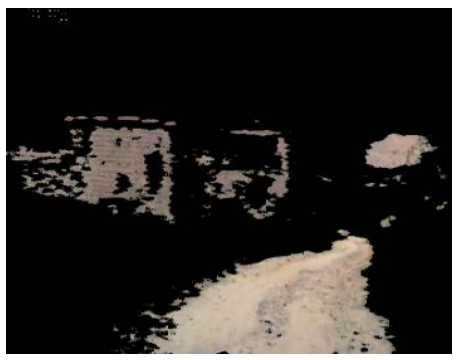

(d) The second class

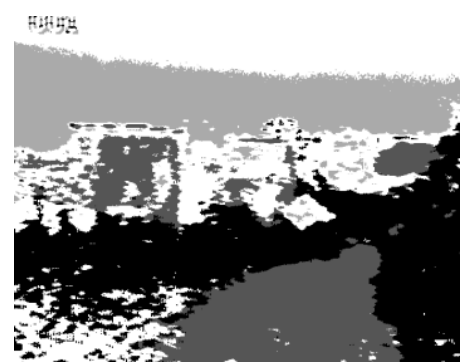

(b) The category graph

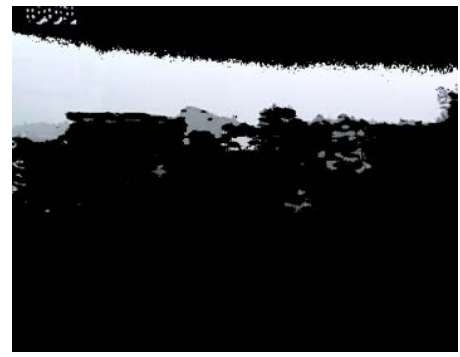

(e) The third class

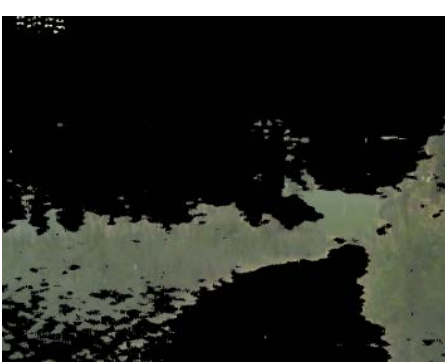

(c) The first class

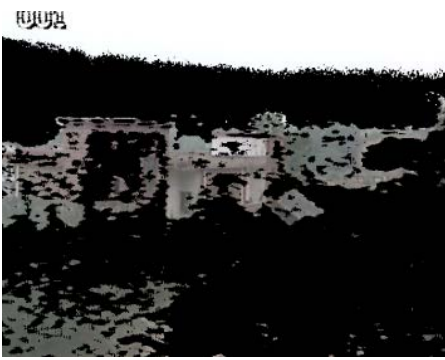

(f) The fourth class

Fig.3. The segmentation result of improved K-means clustering algorithm

\section{Connected Domain Labeling}

Assuming the area in front of the vehicle is road, the central area at the bottom of the image contains the target image segmentation pixels that is used to the subsequent processing. For example, Figure 3(d) is a type of road image. We take connected component labeling algorithm to mark the path of regional after transforming the selected category image into two value image, as shown in Figure 4(a), the interference area are points region and blocks region. The area composed of a small number of pixels and a few pixels can be removed by using area threshold method.

The area threshold is set 50 . The number of the connected area pixels is less than 50 regarded as non-road area is negligible. Connected domain of the central lower part of labeled image is road area as shown in Figure 4(b).

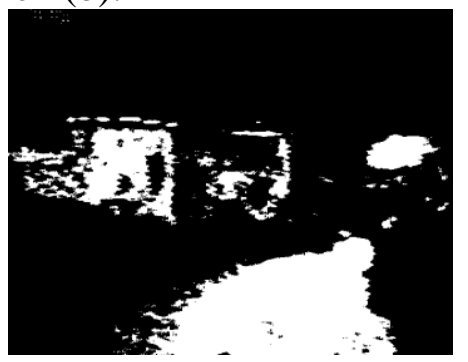

(a)

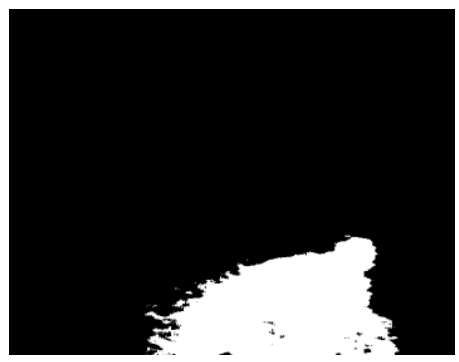

(b)

Fig.4. The results of labeled connected domain

\section{Road Boundary Fitting}

To mark the path area image interlaced sampling, which especially across multiple lines, the line number can be determined by experiment. Line segments represent the pixels belong to the road, as shown in Figure 5(a). The fuzzy of real road boundary leads to the existence of a number of discrete pixels. We ignore the line segments consist of pixels number is less than 15, merge the same row lines whose interval is less than 5 pixels. There are still multiple line segments after processing as shown in Figure 5(b). 

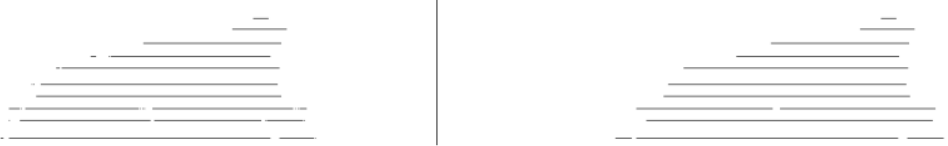

Fig.5. The processing result of sampling line segments

We consider the left endpoints of line segments as the candidate points of the road left boundary, and the right endpoints as the road voted right boundary. The flagged blue points represent the candidate points of the road left boundary and the flagged red points represent the right endpoints as the road voted right boundary, as shown in Figure 6.

There may be some voted road edge points in a row, but the boundary point is only two namely the left and right boundary points. In order to solve this problem, we use a distance discriminant analysis method to select the road boundary points. Taking the road left boundary points for example, the discriminant is as follows:

$$
P^{k}=\operatorname{Arg} \min _{1 \leq i \leq n}\left\|P V_{i}^{k}-P V^{k_{-} \text {closest }}\right\|
$$

In the formula, $P^{k}$ is the road boundary point of the $\mathrm{k}$ row, $P V_{i}^{k}$ is the $i$ th voted edge point in $k$ row, $P V^{k_{\text {cclosest }}}$ is only one road voted edge point that is nearest from the road edge points in $k-1$ and $k+1$ row. $\|\cdot\|$ denotes the Euclidean distance norm, $n$ is the number of boundary points.

The method of road right boundary points selection is the same as above. All boundary points are detected as shown in Figure 7.

Fig.6. Voted edge points of all the line

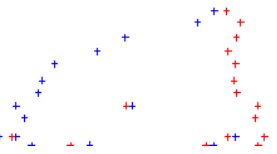

segments

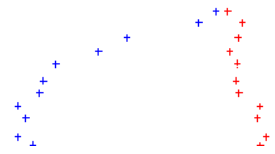

Fig.7. Left edge points and right edge points

Assuming that the direction of the middle line of the road will not suddenly change, the width of the road will not suddenly change. According to the hypothesis, we regard the road boundary point detected as credible. In 2D coordinate system, the width of the lane reduces step by step from the bottom to the top. The graph can clearly show the selected rows of images. The change of the road width is shown in Figure 8(a), the change of coordinate values of the middle points of road boundary in the selected rows is shown Figure 8(b).

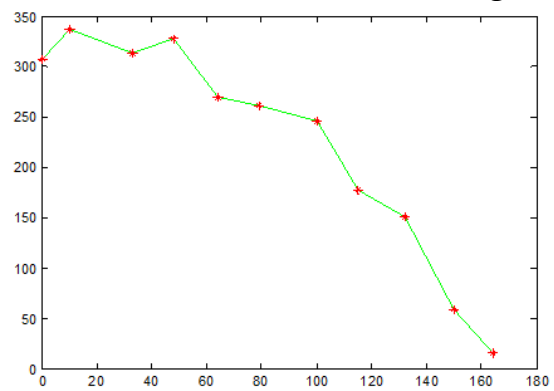

(a)

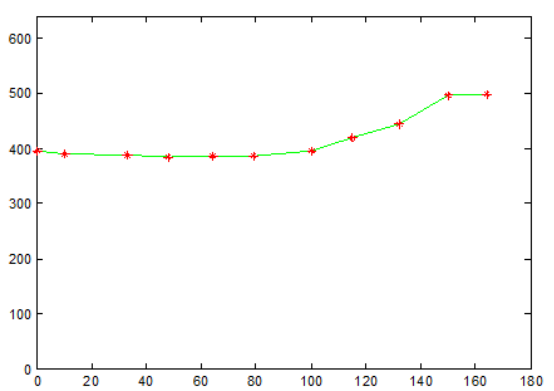

(b)

Fig.8. Statistics of the road width and the coordinates of boundaries' middle point

When the road width or the middle point in selected rows sudden change or are unreasonable, we will ignore the selected line information. So all credible road boundary points obtained as shown in 
Figure 9.

We choose B-splines curve model fitting the reliable edge points and get the optimal control solution using the least squares curve method in order to complete the identification of road boundary. Eight control points $\left(P_{0}, P_{0}, P_{0}, P_{1}, P_{2}, P_{3}, P_{3}, P_{3}\right)$ generates five curve fragments and B-splines curve go through edge points $P_{0} 、 P_{3}$. We consider $P_{0}$ is boundary points at the top of image and $P_{3}$ is the boundary points at the bottom of image. The other control points are acquired according to the difference of the lateral coordinates of the adjacent boundary points.

Assuming $f(x)$ is the initial function, $p(x)$ is a fitting function. $\left(x_{i}, f\left(x_{i}\right)\right)(i=1,2, \ldots, n)$ is a data point). According to the method of least squares, the best fitting curve:

$$
\sum_{i=0}^{n}\left[f\left(x_{i}\right)-p\left(x_{i}\right)\right]^{2} \rightarrow \min
$$

Fitting results of optimal control points of road boundary is shown in Figure 10. On the left boundary, the coordinate values of the initial control points $P i_{1} 、 P i_{2}$ are $(467,330)$, (331, 365), the coordinate values of the optimal control points $P_{1} 、 P_{2}$ are $(459,330) 、(282,365)$. On the right boundary, the coordinate values of the initial control points $P i_{1} 、 P i_{2}$ are $(526,330) 、(509$, 365), the coordinate values of the optimal control points $P_{1} 、 P_{2}$ are $(540,330) 、(490,365)$.

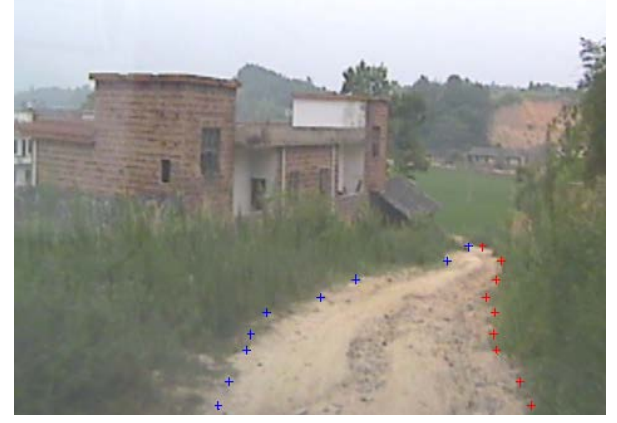

Fig.9. Reliable edge points

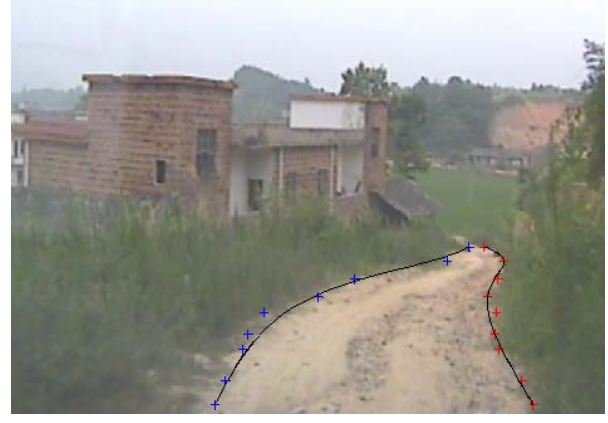

Fig.10. Fitting result

\section{Experimental results and analysis}

Taking AVI video images collected as experimental input data, the format of images is $480 \times 640$. We program the proposed algorithm using $\mathrm{VC}++2005$ on the computer of the frequency of 2.80 GHz Pentium (R) Dual-Core CPU, 2.00GB memory. Experimental results are road identification on color images.

The paper lists the processing results of four pieces of road color images. The vegetation covers most of the regional image in the field road as show in Figure 11(a). The right road boundary covered by the mound in Figure 11(b), so it is difficult to identify the actual boundary. A village road of residual traces of water is shown in Figure 11(c). There is pedestrian interference on road ahead as shown in Figure 11(d). The experimental results show that the proposed algorithm in this paper can identify the road region and has good robustness resisting interference of the vegetation and water mark, obstacles etc.

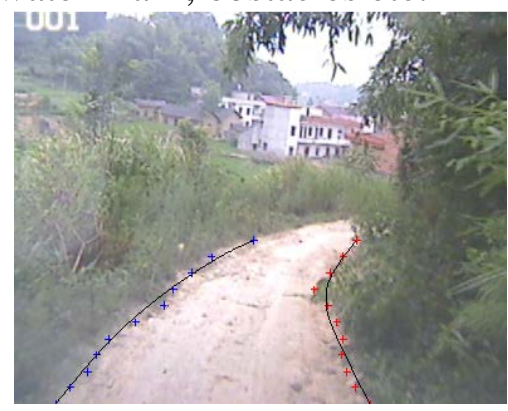

(a)

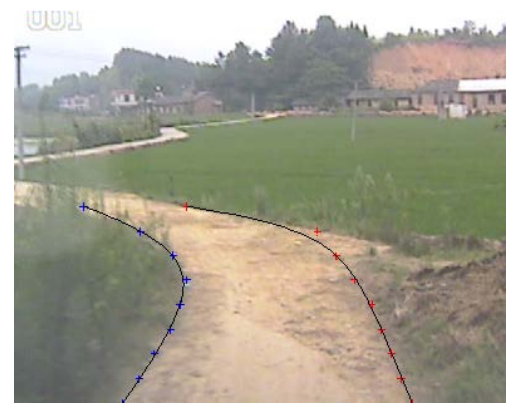

(b) 


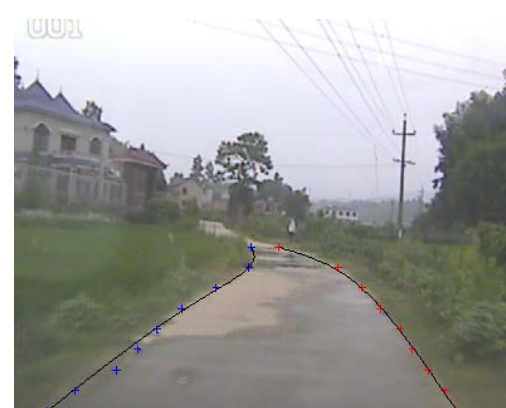

(c)

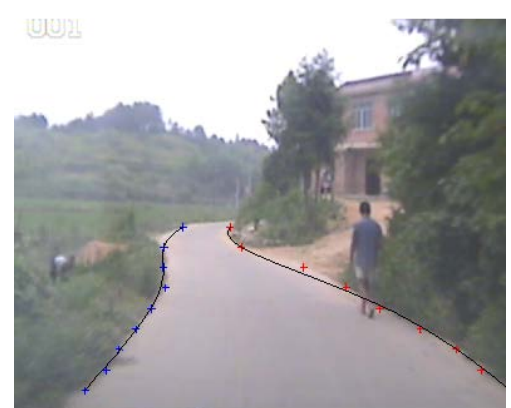

(d)

Fig.11. Experimental result

\section{Conclusion}

This paper proposes the improved K-means clustering algorithm to segment the LAB space, using connected component labeling algorithm to select categories of image region contains Road, interlaced image marking area, determine the trusted path boundary point. The establishment of Bsplines curve model based on searching the optimal control using least square method. The experimental results show that the proposed method based on color segmentation can be used to identify a cross-country road and other unstructured road, finally complete the visual perception of road environment.

\section{References}

[1] Zheng Li, Bin Dai, HanGen He. A Novel Fast Segmentation Method of Unstructured Roads[C]//Proceedings of IEEE Xplore, 2006: 53-56.

[2] Zhu Wen Nan, Chen Qiang, Wang Hong. Lane Detection in Some Complex Conditions[C]//IEEE/RSJ International Conference on Intelligent Robots and Systems, Beijing, China, 2006: 117-122.

[3] Alberto Broggi, Stefano Cattani. An agent based evolutionary approach to path detection for off-road vehicle guidance[J]. Pattern Recognition Letters, 2006, 27(11): 1164-1173.

[4] $\mathrm{Zu}$ Whan Kim. Robust Lane Detection and Tracking in Challenging Scenarios[C]//IEEE Transactions on Intelligent Transportation Systems, NewYork, USA, 2008: 16-26.

[5] Jyun-Guo Wang, Cheng-Jian Lin, Shyi-Ming Chen. Applying Fuzzy Method to Vision-Based Lane Detection and Departure Warning System[J]. Expert Systems with Applications, 2010, 37(7): $113-126$.

[6] Chen Qiang, Wang Hong. A Real Time Lane detection Algorithm Based on a Hyperbola-Hair Model[C]//IEEE Intelligent Vehicles Symposium, Tokyo, Japan, 2006: 510-515.

[7] Su Chung yen, Fa Genhau. An Effective and Fast Lane Detection Algorithm[C]//Proceedings of the 4th International Symposium on Advances in Visual Computing, LasVegas, USA, 2008: 942-948.

[8] Hao Fang, Haifeng Wang, Ze Zhang, Liying Qiu.Vision-based Lane Detection for Mobile Robot Navigation[C]//Proceedings of the 7th World Congress on Intelligent Control and Automation, Chongqing, China, June 25-27, 2008: 3858-3863.

[9] Jingang Huang, Bin Kong, Bichun Li, Fei Zheng. A New Method of Unstructured Road Detection Based on HSV Color Space and Road Features[C]//Proceedings of the 2007 International Conference on Information Acquisition, Jeju City, Korea, July 9-11, 2007: 596-601. 